\title{
Multisystemische Therapie
}

\section{Gregor Berger}

Dr. med., Facharzt für Psychiatrie und Psychotherapie, FMH

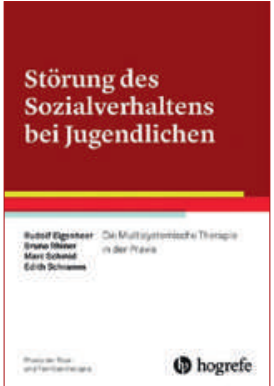

Rudolf Eigenheer, Bruno Rhiner, Marc Schmid und Edith Schramm

Störung des Sozialverhaltens bei Jugendlichen

Die Multisystemische Therapie in der Praxis Reihe: Praxis der Paar- und Familientherapie-Band 10 Göttingen: Hogrefe Verlag; 2016. 289 Seiten, $41.85 \mathrm{CHF}$. ISBN 978-3-8017-2528-0

Dr. med. Gregor Berger Neumünsterallee 3 CH-8032 Zürich gregor.berger[at]puk.zh.ch
Den Autoren ist es gelungen, ein erstes praxisbezogenes und umfassendes deutschsprachiges Werk zur Multisystemischen Therapie für Jugendliche mit einer Störung des Sozialverhaltens zu verfassen, dass 2016 nun in seiner ersten Auflage im Hogrefe Verlag erschienen ist. Etwa 3 von 100 Jugendlichen leiden unter einer Störung des Sozialverhaltens, die häufig mit weiteren Begleiterkrankungen wie Suchtleiden und/oder einer Aufmerksamkeits- und Hyperaktivitätsstörung vergesellschaftet ist. Diese Zielgruppe von Jugendlichen löst in ihren Familien, aber auch im Bildungs-, Sozial- und Gesundheitswesen enorm viel Leid, Arbeit und Kosten aus. Die Multisystemische Therapie (MST Standard) wurde ursprünglich von Scott Henggeler (USA) entwickelt, da herkömmliche Hilfsangebote mit weniger intensiven Behandlungsmodellen scheitern. Bei der MST handelt es sich um eine auf 3 bis 5 Monate zeitlich begrenzte, umfassende und integrative aufsuchende $\mathrm{Ab}$ klärung und Behandlung betroffener Jugendlicher und ihres Umfeldes. Ziel ist es, Veränderungsprozesse von dysfunktionalen Verhaltensweisen zu initiieren und den Jugendlichen und ihre Familien auch nach $\mathrm{Ab}$ schluss der MST-Behandlung Werkzeuge zu vermitteln, die es ihnen ermöglicht, fortschreitende desintegrative Prozesse abzuwenden. Die MST vernetzt sich hierfür mit allen Betroffenen: dem Jugendlichen und seiner Familie, der Schule, Schlüsselfiguren seiner Peergruppe sowie weiteren Bezugspersonen. Ein gemeinsamer Plan zur Behandlung und Unterstützung dieser Problemfamilien wird entwickelt (nach dem FIT-Konzept), um so die Gesundheit der betroffenen Jugendlichen sicherzustellen und zu fördern. Die MST greift hierfür auf evidenzbasierte Erkenntnisse und Arbeitsweisen der kognitiven Verhaltenstherapie und Familien- und Systemtherapie zurück und stellt durch ein engmaschiges Controlling deren Umsetzung sicher. Die Multisystemische Therapie ist einer der am besten evaluierten evidenzbasierten Ansätze mit weltweit über 500 etablierten MST-Teams, die jährlich über $23000 \mathrm{Fa}$ milien behandeln (offizielle Website: www.mstservices. com/teams/team-locations). Dabei konnte die MST auch ausserhalb universitärer Settings erfolgreich implementiert werden und die guten Effizienzdaten kontrollierter Studien in verschiedene Umgebungen übertragen werden.

Das Buch richtet sich an die verschiedene Akteure, die sich mit dieser Zielgruppe auseinandersetzen, wie die
Jugendhilfe, aber auch die Kinder- und Jugendpsychiatrischen Dienste, die Kindes- und Erwachsenenschutzbehörden, die Suchtberatungsstellen, die Schulen und ihre Schulsozialarbeiter, die Jugenddienste der Polizei und schliesslich aber besonders auch an die betroffenen Familien. Das Buch beschreibt in einem ersten Teil die Hintergründe, das Erscheinungsbild und die Konsequenzen der Störung des Sozialverhaltens für die Betroffenen und das System. Im zweiten Teil wird der Multisystemische Ansatz ausführlich und sehr praxisorientiert beschrieben. Im dritten Teil machen die Autoren den Transfer in den therapeutischen Alltag, u.a. auch anhand von praktischen Fallbeispielen. Der vierte Teil des Buches zeigt erste Erfahrungen der ersten MST-Teams der Schweiz im Vergleich zu den internationalen Erfahrungen auf. Im fünften Teil gehen die Autoren auf die Stolpersteine in der Implementierung solcher Therapieangebote ein.

Der kinder- und jugendpsychiatrische Dienst Thurgau leistete hier Pionierarbeit und hat als erster in der Schweiz die MST erfolgreich implementiert und konnte zeigen, dass die Effektivität den guten internationalen Resultanten entspricht. Diese Ergebnisse sind sowohl aus der Perspektive der Eltern, der Betroffenen, aber auch gesellschaftlich und finanziell von hoher Relevanz. In einer Kosteneffektivitäts-Analyse der Thurgauer Stichprobe konnte gezeigt werden, dass die Kosten nur etwa halb so hoch waren (SD 40 bis $61 \%$ ), wie dies bei einer üblichen Behandlung der Fall gewesen wäre. Besonders bemerkenswert in der Thurgauer Studie ist, dass 91\% der Teilnehmer nach sechs Monaten weiterhin in ihren Herkunftsfamilien lebten, $94 \%$ in der Ausbildung verblieben und 91\% keine weiteren Delikte mehr verübt haben. Auch die Folgeuntersuchung nach 18 Monaten zeigte entsprechende Erfolgsquoten von $80 \%, 86 \%$ und $86 \%$. Es ist Bruno Rhiner und seinem Team gelungen, zu zeigen, dass das komplexe MST-Behandlungsprogramm inklusive der hohen Anforderungen an die Umsetzbarkeit auch im schweizerischen Kontext implementiert werden kann und die guten internationalen Ergebnisse repliziert werden konnten.

Zusammenfassend ist dieses Buch für all diejenigen empfehlenswert, die sich mit Jugendlichen mit einer Störung des Sozialverhaltens beschäftigen, und bietet eine wertvolle und praxisrelevante Einführung in die sehr effektive MST. 